University of Nebraska - Lincoln

DigitalCommons@University of Nebraska - Lincoln

Faculty Publications, Department of Psychology

Psychology, Department of

November 2006

\title{
Acculturation status and heavy alcohol use among Mexican American college students: Investigating the moderating role of gender
}

\author{
Byron L. Zamboanga \\ Smith College, bzamboan@smith.edu \\ Marcela Raffaelli \\ University of Nebraska-Lincoln, mraffaelli1@unl.edu \\ Nicholas J. Horton \\ Smith College
}

Follow this and additional works at: https://digitalcommons.unl.edu/psychfacpub

Part of the Psychiatry and Psychology Commons

Zamboanga, Byron L.; Raffaelli, Marcela; and Horton, Nicholas J., "Acculturation status and heavy alcohol use among Mexican American college students: Investigating the moderating role of gender" (2006). Faculty Publications, Department of Psychology. 62.

https://digitalcommons.unl.edu/psychfacpub/62

This Article is brought to you for free and open access by the Psychology, Department of at DigitalCommons@University of Nebraska - Lincoln. It has been accepted for inclusion in Faculty Publications, Department of Psychology by an authorized administrator of DigitalCommons@University of Nebraska - Lincoln. 


\title{
Acculturation status and heavy alcohol use among Mexican American college students: Investigating the moderating role of gender
}

\author{
Byron L. Zamboanga, ${ }^{*}$ Marcela Raffaelli, $\uparrow$ and Nicholas J. Horton $\$$ \\ * Corresponding author; Department of Psychology, Smith College, Northampton, MA 01063 \\ $\uparrow$ Department of Psychology and Institute for Ethnic Studies, University of Nebraska-Lincoln \\ $¥$ Department of Mathematics and Statistics, Smith College
}

\begin{abstract}
We examined whether gender moderates the association between acculturation and heavy alcohol use. The sample consisted of 126 Mexican American college students (Mean age $=24.7$ years; $57 \%$ female) who completed self-report measures of heavy alcohol use, acculturation status (global acculturation and ethnic identity), and relevant control variables (age, peer alcohol use). Multivariable regression revealed that higher levels of ethnic identity were associated with greater frequency of heavy alcohol among men. Conversely, neither measure of acculturation was associated with heavy alcohol use among women. These findings suggest that interventions for Latino/a students should consider the role of culturally relevant variables in heavy alcohol use, particularly for men. They also have implications regarding how acculturation is operationalized in alcohol studies, and suggest directions for future research.
\end{abstract}

Keywords: Acculturation, Alcohol use, Mexican Americans, Gender, College students

Alcohol use is an important health concern among college students (CDC, 1997). Data from national studies (see O'Malley \& Johnston, 2002, for review) indicate that approximately $40 \%$ of college students recently engaged in heavy drinking. Health risks resulting from heavy alcohol use among college students-such as drunk driving, physical fighting, and unsafe sexual behavior-are well-documented in the literature (see Perkins, 2002 for review). Altogether, these findings highlight the need for continued research aimed at identifying key correlates of heavy drinking among college students. 
The drinking patterns of Latinos have received increased attention during the past several years (Nielsen, 2000). (The terms "Hispanic" and "Latino/a" are used in this paper to refer to persons of Mexican, Puerto Rican, Cuban, or other Central American and South American origin living in the U.S.) National surveys with college students indicate that the prevalence of heavy drinking is higher among Latinos compared to Blacks, but lower relative to Whites (O'Malley \& Johnston, 2002). Variations in alcohol use within Latino populations due to age, gender, and Latino sub-group are also evident. For example, one study indicated that college-age (18-25) Latinos have the highest prevalence of heavy drinking (past month) compared to Latinos in other age groups (Ma \& Shive, 2000). National surveys of college students revealed higher prevalence of heavy drinking among Latino males compared to their female counterparts (O'Malley \& Johnston, 2002). Finally, Nielsen (2000) found that Mexican American men reported the highest prevalence of alcohol use compared to men from other Latino groups; among women, the prevalence of alcohol use tended to be higher among Mexican Americans and Puerto Ricans compared to other Latinas. According to Nielsen (2000), the findings regarding Latino group differences in alcohol use (particularly heavy drinking) are generally consistent with those reported in prior studies, suggesting that Mexican Americans (particularly males) may be at risk for elevated alcohol use. Consequently, many studies have been conducted to identify factors that contribute to alcohol use among Mexican Americans.

Investigations of alcohol use among Latinos have highlighted the importance of acculturation (the process of psychological and behavioral adaptation that occurs when two cultures come into contact, as happens when immigrants arrive in a new country or one group is colonized by another; Berry, 1994), and the influence of gender on drinking behaviors (Alaniz et al., 1999 and Caetano \& Clark, 2003). The current study builds on prior literature by examining how gender impacts the relation between acculturation status and heavy alcohol use among Mexican American college students, a growing segment of the U.S. population. It is estimated that within 50 years, nearly $33 \%$ of the under-19 population in the U.S. will be of Hispanic origin or descent (U.S. Department of Health and Human Services, 2001). Thus, information about factors linked to alcohol use among Latino college students could help guide intervention efforts aimed at curbing heavy drinking on college campuses. This study contributes to the mainstream literature aimed at understanding culture-specific correlates of elevated alcohol consumption.

Acculturation has been linked to health risk behaviors such as alcohol use (see Caetano \& Clark, 2003, De La Rosa, 2002 and Epstein et al., 2001 for reviews; for theoretical review of acculturation, see Berry, 2003). However, studies examining the relation between acculturation and alcohol use among Latinos have yielded inconsistent findings; some studies revealed a positive relation, others a negative association, and some no relation (see Caetano \& Clark, 2003, De La Rosa, 2002 and Epstein et al., 2001 for reviews). Thus, the exact role of acculturation in Latino alcohol use remains unclear. There are a few points worth noting regarding the research literature on acculturation and alcohol use among Latinos. First, investigators have relied heavily on proxy indicators such as language use and generation status (De La Rosa, 2002), which do not fully capture the complex, multicultural dimensions of acculturation (e.g., ethnic identification, cultural practices, social relationships). Second, Latinos are culturally heterogeneous and consist of different ethnic subgroups from diverse socioeconomic, historical, and cultural backgrounds; however, studies do not typically take this heterogeneity into account. It has been proposed that in order to better understand the link between acculturation and alcohol use in Latino populations, researchers should examine drinking behaviors in specific Latino groups (Nielsen, 2000) and utilize measures that closely capture the multicultural and psychological dimensions of acculturation status (Marin, Organista, \& Chun, 2003). In the current paper, we focused on one Latino sub-group (Mexican Americans) and used a multidimensional measure of acculturation. 
In addition, we considered another aspect of psychological functioning that has been linked to behaviors and values - ethnic identity, the personal sense of oneself as a member of a particular ethnic group (Phinney, 2003). According to Phinney (2003), ethnic identity is "one aspect of the acculturation process that can be distinguished from other aspects by virtue of its focus on subjective feelings about one's ethnicity" (p. 65). Ethnic identity does not appear to be related to demographic measures of acculturation such as generational status in any consistent manner (Phinney, 2003), which may contribute to the inconsistent findings from prior research on acculturation and alcohol use. In particular, college students may be highly acculturated based on demographic measures such as generation of immigration or language use, yet differ in their adherence to cultural norms, strength of ethnic identification, and other psychological dimensions.

Researchers have highlighted the relevance of cultural identity on substance use (including alcohol use) among Latinos and have advanced hypotheses regarding the link between these variables (Felix-Ortiz \& Newcomb, 1995). One hypothesis contends that highly culturally identified individuals are at risk for substance use because their values and behaviors may conflict with those of the majority society (Felix-Ortiz $\&$ Newcomb, 1995). Such cultural conflicts may give rise to elevated stress, thus increasing their risk for substance use. Conversely, it has been hypothesized that strong identification with one's cultural heritage is linked to positive adjustment. Perhaps highly culturally identified individuals draw support and resource from their cultural networks. In the process of conducting a literature review, research examining the linkages between ethnic and cultural identity and substance use (which includes alcohol) has focused on young adolescents; published articles among Latino college students were nonexistent. To date, studies with Latino and other ethnic minority youth and adults suggest that higher ethnic and cultural identification are associated with decreased substance use (see Burlew, Hucks, Burley, \& Johnson, 2003). However, researchers contend that cultural identity influences substance use but that such relations depend on gender as well as other variables (e.g., familiarity with Latino culture, language use) (Felix-Ortiz \& Newcomb, 1995). Further studies are therefore needed to better understand the link between ethnic identity and alcohol use, as well as factors that moderate the effects of ethnic identity on alcohol use in Latinos. In this study, we examined the potential moderating role of gender on these associations.

Norms regarding drinking behaviors as well as cognitions about the use of alcohol are the product of social learning (Gilbert \& Collins, 1997). Researchers contend that strong sanctions against alcohol use exist for women compared to men in many non-White ethnic groups including Latinos (Gilbert \& Collins, 1997). In one study, Latina adolescents agreed that Latino culture condoned drinking among men, but not women (Flores-Ortiz, 1994). Such cultural norms regarding the use of alcohol suggest that gender may influence the relation between acculturation and drinking behaviors in Latino populations. Conceivably, when Latinas acculturate into a U.S. culture that is less prohibitive (compared to traditional Latino cultures) about the use of alcohol by women, they may modify their drinking behaviors by adopting more liberal attitudes and behaviors toward drinking. In contrast, if there are few cultural sanctions against the use of alcohol by men in more traditional Latino cultures, one might surmise that less acculturated Latino men will engage in more drinking-related behaviors relative to their highly acculturated counterparts.

Consistent with this notion, there is evidence that the association between acculturation and alcohol use in Latinos is influenced by gender (see Alaniz et al., 1999 for review). For example, one study with Latino adults revealed positive associations between acculturation (as measured by language use) and alcohol use for women, but not men (Polednak, 1997). Alaniz et al. (1999) examined the proportion of Mexican American men and women who were drinkers and found that estimates of alcohol use were consistent for men across all acculturation levels; however, for the women, there was a low proportion of drinkers in the less 
acculturated group. The proportion of men and women who were drinkers was similar among those who were more acculturated. The authors also reported positive relations between acculturation and alcohol use for males (as measured by birthplace) and females (as measured by language use). Altogether, these findings highlight the complexities and the role of gender in the relation between acculturation and alcohol use as well as raise questions about the utility of using different indices of acculturation (e.g., birthplace vs. language use) interchangeably in Latino populations.

The current study builds on prior research and addresses a number of methodological and conceptual limitations found in previous studies. To date, the bulk of the literature on acculturation and alcohol use among Latinos has focused primarily on the direct association between these variables. However, this approach is limited; Marin et al. (2003) have urged researchers to view the relation between acculturation and substance use as a complex interaction between individual characteristics and sociocultural factors. Inherent in Latino cultures are gender-specific cultural norms pertaining to alcohol; therefore, the primary goal of this study was to examine how gender moderates the relation between acculturation status and heavy alcohol use in Mexican American college students. We focused on heavy alcohol use because of the high prevalence of drinking and the health-risks associated with elevated usage among college students (O'Malley \& Johnston, 2002). Based on prior research and conceptions of cultural norms and social learning regarding the use of alcohol in Latino cultures, we hypothesized that the relation between acculturation and heavy alcohol use would differ for men and women. Specifically, we expected that more acculturated Mexican American women would engage in heavy alcohol use more frequently than their less acculturated counterparts. Conversely, we anticipated that less acculturated Mexican American men would partake in heavy alcohol use more frequently compared to their more acculturated counterparts.

Researchers have relied heavily on language use and preference, which may represent important aspects of acculturation but do not fully capture other culturally relevant indicators of acculturation (Zane \& Mak, 2003). To address this limitation, we utilized a multidimensional, global index of acculturation and also considered ethnic identity, a psychological aspect of acculturation. Prior literature also suggests that sociodemographic variables such as age (Nielsen, 2000) and peer drinking behavior (Baer, 2002) are linked to alcohol use; thus, we accounted for these variables in our analysis. Finally, many studies combine Latinos into one group and fail to consider the cultural heterogeneity of this population. To address this issue, the analysis was restricted to Mexican Americans.

\section{Method}

\subsection{Participants and procedure}

Participants were drawn from a sample of 18 to 45 year olds $(N=242)$ who self-identified as Latino or Hispanic and participated in a study of Latino family socialization experiences. With the cooperation of four Midwestern post-secondary institutions (two state Universities, < 4\% Hispanic/Latino; one community college, $11 \%$ Hispanic/Latino; and one private college, 3\% Hispanic/Latino), survey packets and reminder postcards were mailed to all currently enrolled Latino/Hispanic students (approximately 30\% response rate). Depending on the institution, respondents either received $\$ 10$ and were entered into a drawing for an additional bonus payment, or were paid $\$ 15$. To ensure student confidentiality, survey packets were direct-mailed by each institution's registration department. 
The current analysis was restricted to 126 Mexican American students. Responses to the ethnic selfidentification and parent ethnicity items on the Multigroup Ethnic Identity Measure (MEIM; Phinney, 1992) were used to identify individuals who self-identified as Mexican, Mexican American, Chicano, and/

or indicated that at least one of their parents was Mexican, Mexican American, or Chicano. The majority of the participants $(86 \%)$ were born in the U.S.

\subsection{Measures}

\subsubsection{Demographics}

Participants reported their age ( $71 \%$ between 18 and 25 years of age) and gender ( $43 \%$ men).

\subsubsection{Heavy alcohol use}

Respondents reported on their frequency of heavy alcohol use (5 or more drinks in one sitting) during the past 30 days; responses ranged from 0 times to 14 or more times. To address the low variability of this item, participants' responses were converted to a four-point scale $(1=$ Never, $2=1$ to 2 times, $3=3$ to 4 times, $4=5$ or more times). About half (51\%) reported heavy alcohol use at least once during the past month. Furthermore, $21 \%$ engaged in heavy drinking one to two times, $13 \%$ reported elevated use three to four times, and $17 \%$ drank heavily five or more times in the past 30 days.

\subsubsection{Peer alcohol use}

We asked respondents to indicate how often their peers drank alcohol using a five-point scale $(1=\mathrm{Nev}$ er drinks, 2 = Seldom drinks, 3 = Sometimes drinks, 4 = Often drinks, $5=$ Always drinks). Approximately $52 \%$ indicated that their peers either often or always drink alcohol, $44 \%$ reported that their peers either seldom or sometimes drinks, and 5\% indicated that their peers never drink.

\subsubsection{Acculturation measures}

We assessed two aspects of acculturation: global acculturation (Acculturation Rating Scale for Mexican Americans-II [ARSMA-II]; Cuellar, Arnold, \& Maldonado, 1995) and ethnic identity (MEIM; Roberts, Phinney, Masse, Chen, \& Roberts, 1999). The ARSMA-II consists of 30 items rated on a five-point scale (1 $=$ Not at all to $5=$ Extremely often or almost always) and encompasses multiple behavioral and attitudinal indicators of acculturation, including language use/preference in different domains (e.g., when speaking, reading, watch TV), ethnic self- and parental-identification preference labels (e.g., My mother identifies or identified herself as "Mexicana"), cultural heritage and ethnic behaviors (e.g., My family cooks Mexican foods), and ethnic interaction (e.g., My friends now are of Mexican origin). The ARSMA-II yields two cultural orientation subscales: Anglo Orientation Scale (AOS; 13 items; $\alpha=.81$ in our study sample) and Mexican Orientation Scale (MOS; 17 items; $\alpha=.88$ in our study sample). Acculturation level is calculated by computing mean scores for each subscale and subtracting the MOS mean from the AOS mean. Cut-off values specified by Cuellar et al. (1995) can be used to classify respondents according to their level of acculturation $(1=$ Very Mexican oriented, $2=$ Mexican oriented to approximately balanced bicultural, $3=$ Slightly Anglo oriented bicultural, $4=$ Strongly Anglo oriented, and $5=$ Very assimilated). Since none of the study participants were classified as "Very Mexican," acculturation level ranged from 2.0 to 5.0. We treated global acculturation scores as a continuous variable in our analysis. 
The MEIM (Phinney, 1992) is a 12-item measure of behavioral and affective aspects of ethnic identity. Statements (e.g., I have a strong sense of belonging to my own ethnic group; I have a lot of pride in my ethnic group and its accomplishments $)$ are rated on a four-point scale $(1=$ Strongly disagree to $4=$ Strongly agree). A total ethnic identity score is derived by reversing negatively worded items and averaging all 12 items ( $\alpha=.90$ in our study sample).

\section{Data analytic approach}

Data analyses were conducted using SPSS version 11.5. First, we examined gender differences and computed bivariate correlations among the study variables, both for the total sample and separately by gender. Second, we conducted multiple linear regression analysis to test the association between two aspects of acculturation status (global acculturation and ethnic identity) and heavy alcohol use and examined whether gender moderates such relations. In our regression model, main effects of background variables (age, gender, and peer alcohol use), acculturation status (global acculturation and ethnic identity), along with acculturation interaction terms with gender (gender $\times$ global acculturation and gender $\times$ ethnic identity) were entered simultaneously. To simplify the interpretation of regression parameters, continuous variables used in the interaction terms were centered prior to computing the interaction terms (Aiken \& West, 1991). Third, we plotted the significant interaction effect using the predicted frequency of heavy alcohol use derived from the model.

\section{Results}

\subsection{Preliminary analyses}

Bivariate correlations and descriptive statistics for the study variables, both for the total sample and separately by gender, are shown in Table 1 . Analysis of variance showed that compared to women, men reported higher frequencies of heavy alcohol use, $F(1,125)=5.62, p<.02, \eta^{2}=.04$ and peer alcohol use, $F(1,125)=6.91, p<.02, \eta^{2}=.05$. Bivariate correlation analyses for the total sample showed that higher frequencies of heavy alcohol use were significantly associated with male gender, younger age, higher peer alcohol use, and higher ethnic identity. Higher peer alcohol use was significantly related to male gender, younger age, and ethnic identity. Ethnic identity was negatively associated with global acculturation. In general, similar bivariate correlation trends emerged for men and women (see Table 1); however, ethnic identity was significantly associated with frequency of heavy alcohol use in men $(r=.28)$ but not women $(r=.01)$.

\subsection{Predicting frequency of heavy alcohol use and the moderating effect of gender}

Results of the multiple linear regression analysis examining predictors of frequency of heavy alcohol use (Table 2) revealed positive associations for ethnic identity and peer alcohol use, and an interaction for gender by ethnicity, $F(7,118)=4.27, p<.001$, model $R^{2}=.20$. Age, gender, global acculturation, and the interaction of gender by global acculturation were not significant contributors (all $p$-values $>.08$ ). To in- 
Horton, Raffaelli, \& Zamboanga in Addictive Behaviors 31 (2006)

Table 1

Bivariate correlations and descriptive statistics for total sample and by gender

\begin{tabular}{llllllll}
\hline Variable & 2 & 3 & 4 & 5 & Mean & SD & Range \\
\hline 1. Age & $-.29 * *$ & -.09 & -.01 & $-.20^{* *}$ & 24.7 & 6.29 & $18-45$ \\
Men & $-.24^{*}$ & $-.19+$ & -.04 & $-.21+$ & 25.7 & 7.43 & $18-45$ \\
Women & $-.43^{* *}$ & .04 & -.01 & $-.25 *$ & 24.0 & 5.22 & $18-41$ \\
2. Peer alcohol use & - & .02 & $.25 * *$ & $.36 * *$ & 3.48 & 1.04 & $1.0-5.0$ \\
Men & - & .11 & $.23 *$ & $.41^{* *}$ & $3.76^{2}$ & .95 & $1.0-5.0$ \\
Women & - & -.05 & $.22^{*}$ & $.28 * *$ & 3.28 & 1.06 & $1.0-5.0$ \\
3. Global acculturation status & - & - & $-.54 * *$ & .01 & 3.59 & .94 & $2.0-5.0$ \\
Men & - & - & $-.58^{* *}$ & .06 & 3.48 & .97 & $2.0-5.0$ \\
Women & - & - & $-.49 * *$ & -.01 & 3.67 & .92 & $2.0-5.0$ \\
4. Ethnic identity & - & - & - & $.16^{*}$ & 3.07 & .59 & $1.3-4.0$ \\
Men & - & - & - & $.28 *$ & 3.15 & .60 & $1.3-4.0$ \\
Women & - & - & - & .01 & 3.00 & .58 & $1.7-3.9$ \\
5. Heavy alcohol use & - & - & - & - & 2.00 & 1.14 & $1.0-4.0$ \\
Men & - & - & - & - & $2.24^{\mathrm{b}}$ & 1.21 & $1.0-4.0$ \\
Women & - & - & - & - & 1.76 & 1.04 & $1.0-4.0$ \\
\hline
\end{tabular}

$N=126$ (54 men; 72 women). Significant correlations (one-tailed): $* * p<.01, * p<.05,+p<.10$. Significant gender differences in means: ${ }^{\mathrm{a}} p<.02$, partial $\eta^{2}=.04 ;{ }^{\mathrm{b}} p<.02$, partial $\eta^{2}<.05$.

terpret the gender by ethnic identity interaction, we plotted the predicted values of frequency of heavy alcohol use as a function of gender and centered ethnic identity scores ranging from -1 to 1 . As can be seen in Fig. 1, there was a positive association between ethnic identity score and frequency of heavy alcohol use for men, but little or no association for women.

\section{Discussion}

The prevalence of heavy drinking on college campuses highlights the need for continued research on alcohol use among young adults in the U.S. Our study sought to better understand culture-specific correlates of heavy alcohol use among Mexican American college students, who represent a growing segment of the U.S. population. We built on prior research in four main ways in an attempt to clarify inconsistent results from past research on acculturation and alcohol use in Latino populations. First, we focused on heavy alcohol use, which poses a serious risk to college students (O’Malley \& Johnston, 2002). Second, our

Table 2

Multiple linear regression analysis predicting frequency of heavy alcohol use

\begin{tabular}{lccc}
\hline Variable & $B$ & SE $B$ & $\beta$ \\
\hline Age & -.02 & .02 & -.11 \\
Gender & -.35 & .20 & -.15 \\
Peer alcohol use & .29 & .10 & $.26 * *$ \\
Global acculturation status & .26 & .19 & .21 \\
Gender $\times$ global acculturation status & -.30 & .24 & -.19 \\
Ethnic identity & .69 & .30 & $.36 *$ \\
Gender $\times$ ethnic identity & -.81 & .39 & $-.31 *$ \\
\hline
\end{tabular}

$N=126$, Model $R^{2}=20 . * *<.01, * p<.05$. Age, peer alcohol use, ethnic identity, and global acculturation status were centered prior to computing the interaction terms. ${ }^{2}$ Coded as $M e n=0$ and Women $=1$. 


\section{B.L. Zamboanga et al. / Addictive Behaviors 31 (2006) 2188-2198}

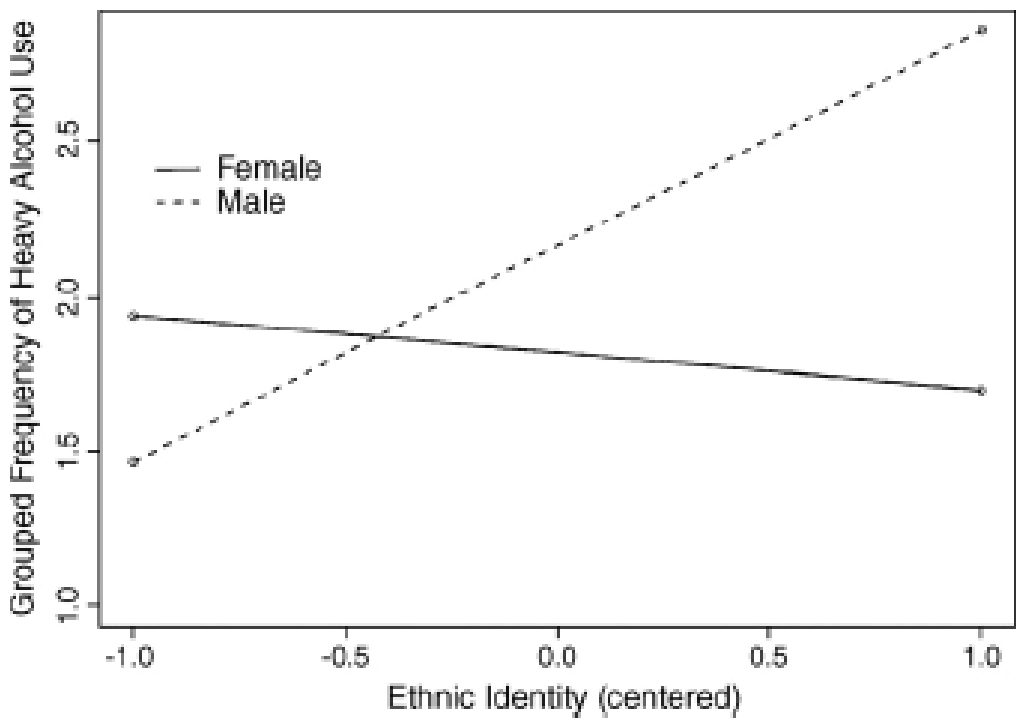

Fig. 1. Plot of the ethnic identity $\times$ frequency of heavy alcohol use interaction.

study included two indices of acculturation, including a multidimensional measure that indexed primarily behavioral aspects of acculturation (e.g., language use and preference, cultural behavior, social ties), and a measure of ethnic identity, a psychological aspect of acculturation. Third, in recognition of the within-group diversity of the Latino population, we limited our college sample to Mexican Americans. Finally, consistent with recent recommendations that researchers investigate potential moderators of the association between acculturation and substance use (Marin et al., 2003), we examined the moderating role of gender.

For the male Mexican American college students in our sample, we found a significant positive association between ethnic identity and frequency of heavy alcohol use. That is, men who were more ethnically identified also tended to report more frequent heavy alcohol use, after other relevant variables (e.g., age, peer alcohol use, global acculturation status) were taken into account. In contrast, there was minimal association between ethnic identity and frequency of heavy alcohol use for women. The findings for men are consistent with the hypothesis that identification with Latino culture is associated with elevated alcohol use. An alternate possibility is that highly ethnically identified individuals consume higher levels of alcohol because their values and behaviors may conflict with those of the majority society. We could not examine this in our study, but future studies could examine the degree to which cultural conflicts and adjustment difficulties mediate and/or moderate the relation between acculturation and alcohol use in Latino men. The lack of association between global acculturation and alcohol use supports the notion that researchers should consider moving from measuring external aspects of acculturation (e.g., language use and related-behaviors) to focusing on psychological dimensions (Zane \& Mak, 2003). In short, future research on Mexican American college students' alcohol use can build on these findings by teasing out what aspects of psychological acculturation are linked to alcohol use, perhaps by examining norms and values directly and examining culture-specific norms pertaining to alcohol use. 
In light of the findings noted above, there are a few considerations worth noting with respect to social influences and their relevance to acculturation and college drinking among Mexican American college students. Social norms can influence the extent to which drinking will be encouraged (Maisto, Carey, \& Bradizza, 1999). Norms can be learned through observation of socializing agents such as peers during college. The college years can be characterized as a time of increased social activity during which alcohol use is highly likely to occur (Baer, 2002). Increased exposure to parties that involve drinking and the presence of a Greek system can facilitate elevated use during the college years. Indeed, national reports indicate higher prevalence rates of alcohol use among college students compared to their age-mates who are not in college (O’Malley \& Johnson, 2002). Conceivably, less acculturated, Mexican American college men may be at risk for elevated use, particularly in social settings (i.e., college) where opportunities and peer influences to consume alcohol are likely to be quite high. Clearly, further research designed to investigate how the college environment might impact acculturation and drinking-related behaviors in Mexican American student populations and other Latino subgroups are needed.

The lack of association between the study variables and heavy alcohol use in our sample of Mexican American college women contradicts prior research showing positive associations between acculturation and alcohol use among Latinas (e.g., Alaniz et al., 1999 and Polednak, 1997). There are several differences between the current study and past research that may account for the discrepancy in findings. Our study employed two multidimensional measures of acculturation, whereas prior research has typically relied on language use or birthplace as proxy indicators of acculturation. This investigation also focused on heavy drinking ( $5+$ drinks in one sitting) rather than alcohol use (frequency/quantity) in general and our female sample consisted primarily of young women (72\% ages 18-25) attending college. Further studies are needed in order to better understand what acculturative factors (if any) influence different aspects of alcohol use among Mexican American college women.

There were some limitations worth noting in our investigation. The cross-sectional study design precludes any inferences of causality regarding the relation between ethnic identity and heavy alcohol use. Second, our study focused on Mexican American college students; therefore, caution must be used when generalizing our findings to Mexicans and Mexican Americans who do not attend (or have not attended) college. Furthermore, Latino subgroups differ on many sociocultural variables such as history and length of stay in the U.S., socioeconomic status, and visible-minority status (Suarez-Orozco \& Paez, 2002); therefore, it remains unclear whether or how the current findings would have differed had we used a nationally representative sample of Latinos. Third, we relied on self-reported alcohol use so it is possible that respondents may have under- or over-reported their drinking behaviors. Finally, the modest response rate $(30 \%)$, although comparable to that obtained in other mail-based surveys (e.g., Kaplowitz, Hadlock, \& Levine, 2004), may have biased the sample in unknown ways. Future research should therefore be mindful of strategies that facilitate increase response rates when conducting these types of studies (see Edwards, et al., 2002). In light of these sample considerations, it should be noted that the proportion of respondents who indicated heavy alcohol use was comparable to national studies with college students. The prevalence of heavy alcohol use among college students is estimated at 40\% (O'Malley \& Johnston, 2002). Prevalence rates of heavy alcohol use among college students are particularly high for Whites ( $40 \%$ to $50 \%$ ), followed by Hispanics (30\% to $40 \%$ ), then Blacks (15\% to $25 \%$ ). In the present study, half of the participants reported current heavy episodic alcohol use at least once. Hence, compared to national college samples, there were comparable percentages in the proportion of students in this study who reported heavy alcohol use. 
Despite the study limitations, the current findings have potential implications for prevention efforts, theory refinement, and future research. Intervention programs aimed at reducing heavy alcohol use among Mexican American college students might consider gender socialization differences regarding attitudes toward alcohol use and explicitly address the moderating role of gender. It is possible that intervention programs designed to combat heavy alcohol use that include encouraging or strengthening ethnic identity may not be optimal for Mexican American male students. From a theoretical perspective, the current study highlights the relevance of various aspects of acculturation and raises questions about the appropriateness of using different measures of acculturation (e.g., global acculturation vs. ethnic identity) interchangeably in research with Mexican American college students as well as non-Mexican Latino students (Raffaelli, Zamboanga, \& Carlo, 2005). Finally, this investigation sheds light on future research directions aimed at gaining a full understanding of the complex relation between acculturation and alcohol use in Mexican American college students and possibly other Latino student subgroups as well.

\section{Acknowledgements}

This project was supported by grants to Marcela Raffaelli from the National Institute of Mental Health and the Office of the Research Council, University of Nebraska-Lincoln. The authors are thankful to Kevin Berryman, Derek Bothern, Erin Fling, Elizabeth Johnson, Craig Peters, Melissa VanDyke, Shana Weiseler, and Paula Womack for their assistance with this project. Special thanks to Sherill Pineda and Sherry Wang for their editorial comments on this manuscript.

\section{References}

Aiken \& West, 1991.- L.S. Aiken and S.G. West, Multiple regression: Testing and interpreting interactions, Sage, Newbury Park, CA (1991).

Alaniz et al., 1999.- M.L. Alaniz, A.J. Treno and R.F. Saltz, Gender, acculturation, and alcohol consumption among Mexican Americans, Substance Use and Misuse 34 (1999), pp. 1407-1426.

Baer, 2002.- J.S. Baer, Student factors: Understanding individual variation in college drinking, Journal of Studies on Alcohol 14 (2002), pp. 40-53

Berry, 2003.- J.W. Berry, Conceptual approaches to acculturation. In: K.M. Chun, P.B. Organista and G. Marín, Editors, Acculturation: Advances in theory, measurement, and applied research, American Psychological Association, Washington, DC (2003), pp. 17-38.

Berry, 1994.- J.W. Berry, Acculturative stress. In: W.J. Lonner and R.S. Malpass, Editors, Psychology and culture, Allyn and Bacon, Boston, MA (1994), pp. 211-215.

Burlew et al., 2003.- A.K. Burlew, T. Hucks, R. Burley and C. Johnson, Prevention of substance abuse among ethnic minority youth. In: G. Bernal, J.E. Trimble, A.K. Burley and F.T.L. Leong, Editors, Handbook of racial and ethnic minority psychology, Sage, Thousand Oaks (2003), pp. 633-655.

Caetano \& Clark, 2003.- R. Caetano and C.L. Clark, Acculturation, alcohol consumption, smoking, and drug use among Hispanics. In: K.M. Chun, P.B. Organista and G. Marín, Editors, Acculturation: Advances in theory, measurement, and applied research, American Psychological Association, Washington, DC (2003), pp. 223-239.

Center for Disease Control, 1997.- Center for Disease Control, Youth risk behavior surveillance: National college health risk behavior survey-United States, MMWR 46 (1997), p. SS-6.

Cuellar et al., 1995. - I. Cuellar, B. Arnold and R. Maldonado, Acculturation rating scale for Mexican Americans-II: A revision of the original ARSMA scale, Hispanic Journal of Behavioral Sciences 17 (1995), pp. 275-304.

De La Rosa, 2002.- M. De La Rosa, Acculturation and Latino adolescents' substance use: A research agenda for the future, Substance Use and Misuse 37 (2002), pp. 429-456. 
Horton, Raffaelli, \& Zamboanga in Addictive Behaviors 31 (2006)

Edwards et al., 2002.- P. Edwards, I. Roberts, M. Clarke, C. DiGuiseppi, S. Pratap and R. Wentz et al., Increasing response rates to postal questionnaires: Systematic review, British Medical Journal 324 (2002), pp. 1183-1185.

Epstein et al., 2001.- J.A. Epstein, G.J. Botvin and T. Diaz, Linguistic acculturation associated with higher marijuana and polydrug use among Hispanic adolescents, Substance Use and Misuse 36 (2001), pp. 477-499.

Felix-Ortiz \& Newcomb, 1995.- M. Felix-Ortiz and M. Newcomb, Cultural identity and drug use among Latino and Latina adolescents. In: G. Botvin, S. Schinke and M. Orlandi, Editors, Drug abuse prevention with multiethnic youth, Sage, Thousand Oaks (1995), pp. 147-165.

Flores-Ortiz, 1994. - Y.G. Flores-Ortiz, The role of cultural and gender values in alcohol use patterns among Chicana/Latina high school and university students: Implications for AIDS prevention, International Journal of the Addictions 29 (1994), pp. 1149-1171.

Gilbert \& Collins, 1997.- J.M. Gilbert and R.L. Collins, Ethnic variation in women's and men's drinking. In: R.W. Wilsnack and S.C. Wilsnack, Editors, Gender and alcohol: Individual and social perspectives, Rutgers Center of Alcohol Studies, New Brunswick (1997), pp. 357-378.

Kaplowitz et al., 2004.- M.D. Kaplowitz, T.D. Hadlock and R. Levine, A comparison of web and mail survey response rates, Public Opinion Quarterly 68 (2004), pp. 94-101.

Ma and Shive, 2000.- G.X. Ma and S. Shive, A comparative analysis of perceived risks and substance use among ethnic groups, Addictive Behaviors 25 (2000), pp. 361-371.

Maisto et al., 1999.- S.A. Maisto, K.B. Carey and C.M. Bradizza, Social Learning Theory. In: M.S. Goldman, F.K. Del Boca and J. Drakes, Editors, Psychological theories of drinking and alcoholism (2nd), Guilford, New York (1999), pp. 203-245.

Marin et al., 2003.- G. Marin, P.B. Organista and K.M. Chun, Acculturation research: Current issues and findings. In: G. Bernal, J.E. Trimble, A.K. Burley and F.T.L. Leong, Editors, Handbook of racial and ethnic minority psychology, Sage, Thousand Oaks (2003), pp. 208-219.

Nielsen, 2000.- A.L. Nielsen, Examining drinking patterns and problems among Hispanic groups: Results from a national survey, Journal of Studies on Alcohol 61 (2000), pp. 301-310.

O’Malley \& Johnston, 2002.- P.M. O’Malley and L.D. Johnston, Epidemiology of alcohol and other drug use among American college students, Journal of Studies on Alcohol 14 (2002), pp. 23-39.

Perkins, 2002.- H.W. Perkins, Surveying the damage: A review of research on consequences of alcohol use misuse in college populations, Journal of Studies on Alcohol 14 (2002), pp. 91-100.

Phinney, 2003.- J.S. Phinney, Ethnic identity and acculturation. In: K.M. Chun, P.B. Organista and G. Marín, Editors, Acculturation: Advances in theory, measurement, and applied research, American Psychological Association, Washington, DC (2003), pp. 63-81.

Phinney, 1992. - J.S. Phinney, The multigroup ethnic identity measure: A new scale for use with diverse groups, Journal of Adolescent Research 7 (1992), pp. 156-176.

Polednak, 1997.- A.P. Polednak, Gender and acculturation in relation to alcohol use among Hispanic (Latino) adults in two areas of the Northwestern United States, Substance Use and Misuse 32 (1997), pp. 1513-1524.

Raffaelli et al., 2005.- M. Raffaelli, B.L. Zamboanga and G. Carlo, Acculturation status and sexuality among female Cuban-American college students, Journal of American College Health 54 (2005), pp. 7-13.

Roberts et al., 1999.- R.E. Roberts, J.S. Phinney, L.C. Masse, Y.R. Chen and C.R. Roberts, The structure of ethnic identity of young adolescent from diverse ethnocultural groups, Journal of Early Adolescence 19 (1999), pp. $301-322$.

Suárez-Orozco \& Páez, 2002.— In: M.M. Suárez-Orozco and M.M. Páez, Editors, Latinos: Remaking America, University of California Press, Berkeley (2002).

U.S. Department of Health and Human Services, 2001._ U.S. Department of Health and Human Services, Mental health: Culture, race and ethnicity - supplement to mental health: A report of the surgeon general. Rockville, MD (2001).

Zane \& Mak, 2003.- N. Zane and W. Mak, Major approaches to the measurement of acculturation among ethnic minority populations: A content analysis and an alternative empirical strategy. In: K.M. Chun, P.B. Organista and G. Marín, Editors, Acculturation: Advances in theory, measurement, and applied research, American Psychological Association, Washington, DC (2003), pp. 39-60. 